\title{
Effect of Core Nanostructure on the Thermomechanical Properties of Soft Nanoparticles
}

Ernesto Rafael Osorio-Blanco, ${ }^{\dagger}$ Julian Bergueiro, ${ }^{\dagger}$ Bilen Emek Abali, ${ }^{\perp}$ Svenja Ehrmann, ${ }^{\dagger}$ Christoph Böttcher, ${ }^{\S}$ Alejandro J. Müller, ${ }^{ \pm \phi}$ José Luis Cuéllar-Camacho* ${ }^{* \dagger}$ and Marcelo Calderón $* \Vdash \phi$

$\dagger$ Freie Universität Berlin, Institute of Chemistry and Biochemistry, Takustr. 3, 14195 Berlin, Germany

$\perp$ Technische Universität Berlin, Institute of Mechanics, Einsteinufer 5, 10587 Berlin, Germany

$\S$ Freie Universität Berlin, Research Center of Electron Microscopy and Core Facility BioSupraMol, Institute of Chemistry and Biochemistry, Fabeckstr. 36a, 14195 Berlin, Germany

\pm POLYMAT and Polymer Science and Technology Department, Faculty of Chemistry, University of the Basque Country UPV/EHU, Paseo Manuel de Lardizabal 3, 20018 Donostia-San Sebastián, Spain

†OLYMAT and Applied Chemistry Department, Faculty of Chemistry, University of the Basque Country, UPV/EHU, Paseo Manuel de Lardizabal 3, 20018 Donostia-San Sebastián, Spain

$\phi I K E R B A S Q U E$, Basque Foundation for Science, 48013 Bilbao, Spain 


\section{Materials}

Ammonia solution (Roth, 25\%), bisacrylamide (BIS; Sigma-Aldrich), ethanol (dry) (Acros Organics, 99.8\%), potassium peroxodisulfate (KPS; Merck), sodiumdodecylsulfate (SDS; Sigma-Aldrich) sodium hydroxide $(\mathrm{NaOH}$; Merck Millipore), tetraethylorthosilicate (TEOS; Sigma-Aldrich), 3-(trimethoxysilyl) propyl methacrylate (TPM; Sigma-Aldrich) were used without further purification. The monomer N-isopropylacrylamide (NIPAM; Sigma-Aldrich) was purified by recrystallization from hexane (Sigma-Aldrich). The water used during the project was purified using a Milli-Q system (Millipore).

\section{Methods}

Reactions under oxygen- and water-free conditions were performed under an argon inert gas atmosphere, using standard Schlenk techniques. Reagents and solvents were added with syringes via septum under argon flow. Solids were added as a solution or as powder under argon flow. The characterization of the nanoarchitectures was performed by ${ }^{1} \mathrm{H}-\mathrm{NMR}, \mathrm{IR}$, and UV. Size and polydispersity were analyzed by DLS, NTA, TEM, SEM, and AFM.

Proton nuclear magnetic resonance $\left({ }^{1} \mathbf{H}-\mathbf{N M R}\right)$ : The spectra were recorded with Jeol ECX $(400 \mathrm{MHz})$ at concentrations between $5-20 \mathrm{mg} / \mathrm{mL}$. The samples were dissolved in deuterated solvents $\left(\mathrm{D}_{2} \mathrm{O},{ }^{1} \mathrm{H}: 4.79 \mathrm{ppm} ; \mathrm{CDCl}_{3},{ }^{1} \mathrm{H}: 7.26 \mathrm{ppm}\right)$. The results were analyzed using MestReNova (version 7.1.2). The multiplicities are listed as follow: $\mathrm{s}$ (singlet), $\mathrm{d}$ (doublet), $\mathrm{t}$ (triplet), q (quartet), $\mathrm{m}$ (multiplet).

Fourier transform infra-red spectroscopy (FT-IR): FT-IR spectra were recorded on a Jasco FT/IR-410 spectrometer (4000-500 $\left.\mathrm{cm}^{-1}\right)$ and analyzed using Omnic software.

Dynamic light scattering (DLS): Size distribution was measured at various temperatures by dynamic light scattering (DLS) using a Zetasizer Nano-ZS 90 (Malvern, UK) equipped with a He-Ne laser $(\lambda=633 \mathrm{~nm})$ under a scattering angle of $173^{\circ}$. For equilibration, samples with a concentration of $1 \mathrm{mg} / \mathrm{mL}$ were incubated at the desired temperature for 5 min prior to the measurement. Particle size distributions are given as the average of three measurements from intensity distribution curves. Due to the monomodal distribution as well as single exponential decay of the autocorrelation functions of the samples, the hydrodynamic diameters are reported from the intensity distribution curves.

Nanoparticle tracking analysis (NTA): Nanoparticle size and concentration were measured by NTA using a Nanosight NS500 (Malvern Instruments, Malver, UK). Nanoparticle concentrations of $1 \mu \mathrm{g} / \mathrm{mL}$ were used for the analysis. Particle sizes and concentrations are given as the average of three measurements.

Synthesis and characterization of thermoresponsive nanoarchitectures (NGs, $\mathrm{SiO}_{2} @ \mathrm{NGs}$, and NCs)

Synthesis and characterization of PNIPAM-BIS nanogels (NGs): NGs were synthesized using a precipitation polymerization technique. NIPAM $(100 \mathrm{mg})$ was dissolved together with BIS (7.8 mg) and SDS (2 mg) in Milli Q water $(5 \mathrm{~mL})$. The solution was purged with Argon for $1 \mathrm{~h}$ and then heated up to $70^{\circ} \mathrm{C}$. The reaction was started by adding a solution of KPS in water ( $2 \mathrm{mg}$ dissolved in $1 \mathrm{~mL}$ water) to the reaction mixture. The polymerization was stirred for $4 \mathrm{~h}$ at $70{ }^{\circ} \mathrm{C}$ and subsequently dialyzed for $5 \mathrm{~d}$. The product was lyophilized and kept in the fridge for further analysis via ${ }^{1} \mathrm{H}-\mathrm{NMR}$, TEM, AFM and DLS. Yield: quantitative. ${ }^{1} \mathrm{H}-\mathrm{NMR}$ (400 MHz, $\mathrm{D}_{2} \mathrm{O}$ ); $\delta: 1.19$ (s, $6 \mathrm{H}$, isopropyl groups of NIPAM), 1.62 (br, $2 \mathrm{H}$, polymer backbone), 2.15 (br, $1 \mathrm{H}$, polymer backbone), 3.92 (br, $1 \mathrm{H}$, isopropyl group NIPAM). 


\section{NGs}
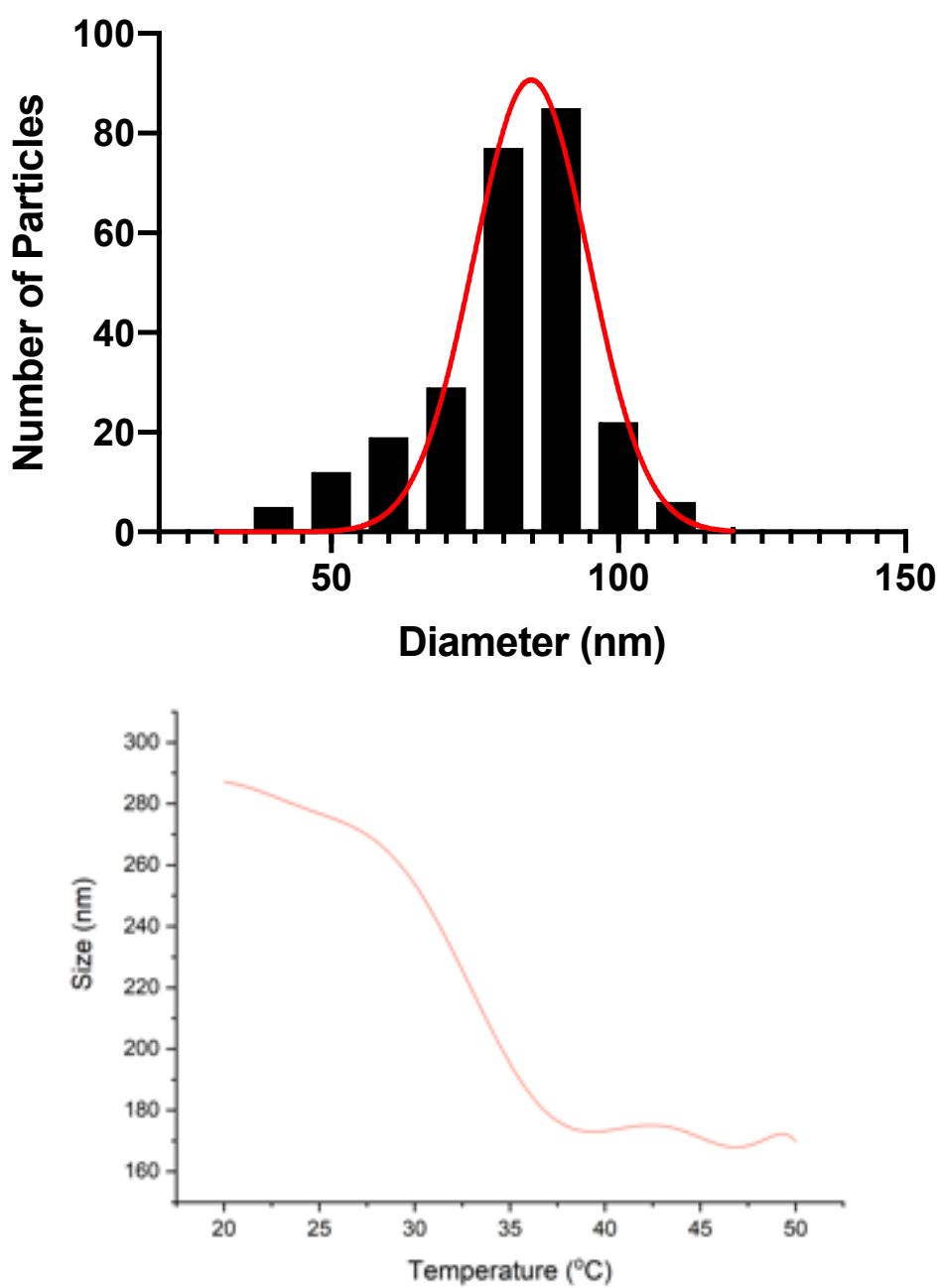

Figure 1. Size versus Temperature trend.

Synthesis and characterization of nanogels with silica nanoparticles in their core (SiO $@ N G s):$ Silica nanoparticles $\left(\mathrm{SiO}_{2} \mathrm{NPs}\right)$ were synthesized using the Stöber method. A mixture of $11 \mathrm{~mL}$ ammonia and $130 \mathrm{~mL}$ extra pure ethanol was heated up to $60{ }^{\circ} \mathrm{C}$ in a $300 \mathrm{~mL}$ three-neck round flask equipped with a reflux condenser and a magnetic stirrer. ${ }^{1}$ After 60 minutes equilibration time, a preheated solution of TEOS $(5 \mathrm{~mL})$ in ethanol $(15 \mathrm{~mL})$ was rapidly added. The reaction mixture became turbid within 30 minutes after the addition. The reaction was stirred for 24 hours at a constant temperature of $60{ }^{\circ} \mathrm{C}$. Afterwards the mixture was cooled down to room temperature and TMP (5.3982 x $10^{-18} \mathrm{~g}$ TMP per $\left.\mathrm{SiO}_{2} \mathrm{NP}\right)$ was added dropwise. The dispersion was stirred for 12 hours at room temperature and then refluxed for 1 hour. Finally, the $\mathrm{SiO}_{2} \mathrm{NPs}$ were purified by centrifugation (3 cycles of $6000 \mathrm{rpm}, 1$ hours) and redispersed in fresh ethanol to yield a dispersion with a concentration of $0.045 \mathrm{~g} \mathrm{~mL}^{-1}$. The characterization of the product was done via IR analysis. Yield: $100 \%$; IR $\left(\mathrm{cm}^{-1}\right): 1733(\mathrm{C}=\mathrm{O})$, $1652(\mathrm{C}=\mathrm{C}), 945$ (Si-O-Si), $791(\mathrm{Si}-\mathrm{OH})$.

The synthesis of $\mathrm{SiO}_{2} @ \mathrm{NGs}$ was performed following a previously reported seeded precipitation polymerization methodology. ${ }^{2}$ NIPAM $(100 \mathrm{mg})$ was dissolved together with BIS $(7.8 \mathrm{mg})$ and SDS $(2 \mathrm{mg})$ in Milli Q water $(5 \mathrm{~mL})$. The solution was purged with Argon for $1 \mathrm{~h}$ and then heated up to $70{ }^{\circ} \mathrm{C}$. After 30 minutes equilibration time, a solution of modified silica NPs (10 mg) with a concentration of $45 \mathrm{mg} / \mathrm{mL}$ was added dropwise. The reaction was started by adding KPS ( $2 \mathrm{mg}$ dissolved in $1 \mathrm{~mL}$ water) to the solution, becoming turbid within ten minutes. The reaction was stirred for $4 \mathrm{~h}$ at $70{ }^{\circ} \mathrm{C}$. The product was purified by centrifugation 
and redispersion in fresh Milli Q water and afterwards lyophilized and kept in the fridge. The characterization of the product was done via ${ }^{1} \mathrm{H}-\mathrm{NMR}$, TEM, AFM and DLS. Yield: $65 \% .{ }^{1} \mathrm{H}-$ NMR (400 MHz, $\left.\mathrm{D}_{2} \mathrm{O}\right) ; \delta: 1.14$ (s, $6 \mathrm{H}$, isopropyl groups of NIPAM), 1.57 (br, $2 \mathrm{H}$, polymer backbone), 1.95 (br, $1 \mathrm{H}$, polymer backbone), 3.89 (br, $1 \mathrm{H}$, isopropyl group NIPAM).

\section{$\mathrm{SiO}_{2} \mathrm{NGs}$}
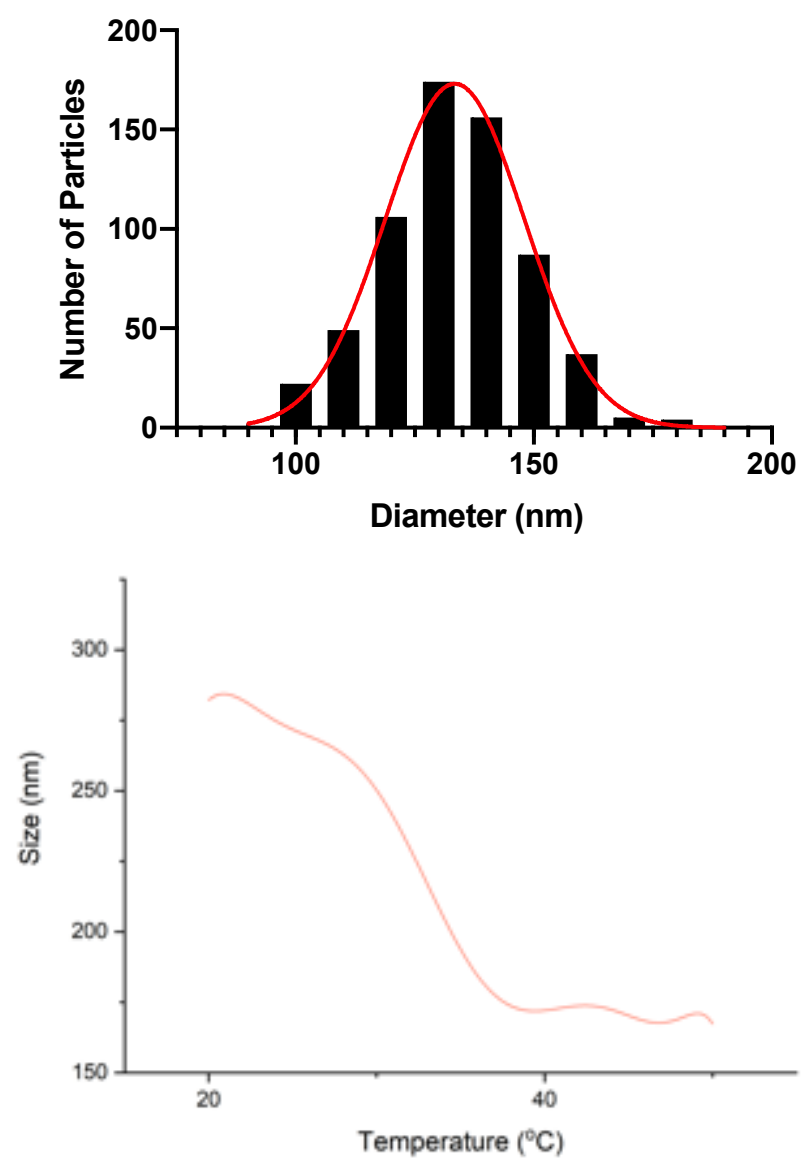

Figure 2. Size versus Temperature trend.

Synthesis and characterization of PNIPAM-BIS nanocapsules (NCs): PNIPAM-BIS $\mathrm{SiO}_{2} @ \mathrm{NGs}(10 \mathrm{mg})$ were dissolved in $20 \mathrm{~mL}$ of $\mathrm{NaOH}(0.05 \mathrm{M})$. The solution was stirred for 4 days at r.t. Finally, the NCs were purified by dialysis in water until a neutral $\mathrm{pH}$ was achieved. Subsequently, the NCs were lyophilized and stored in the fridge. The characterization of the product was done via ${ }^{1} \mathrm{H}-\mathrm{NMR}$, TEM, AFM and DLS. Yield: $100 \% ;{ }^{1} \mathrm{H}-\mathrm{NMR}\left(400 \mathrm{MHz}, \mathrm{D}_{2} \mathrm{O}\right)$; $\delta: 1.18$ (s, $6 \mathrm{H}$, isopropyl groups of NIPAM), 1.59 (br, $2 \mathrm{H}$, polymer backbone), 1.95 (br, $1 \mathrm{H}$, polymer backbone), 3.91 (br, $1 \mathrm{H}$, isopropyl group NIPAM). The core dissolution was confirmed using TEM and AFM. 


\section{NCs}
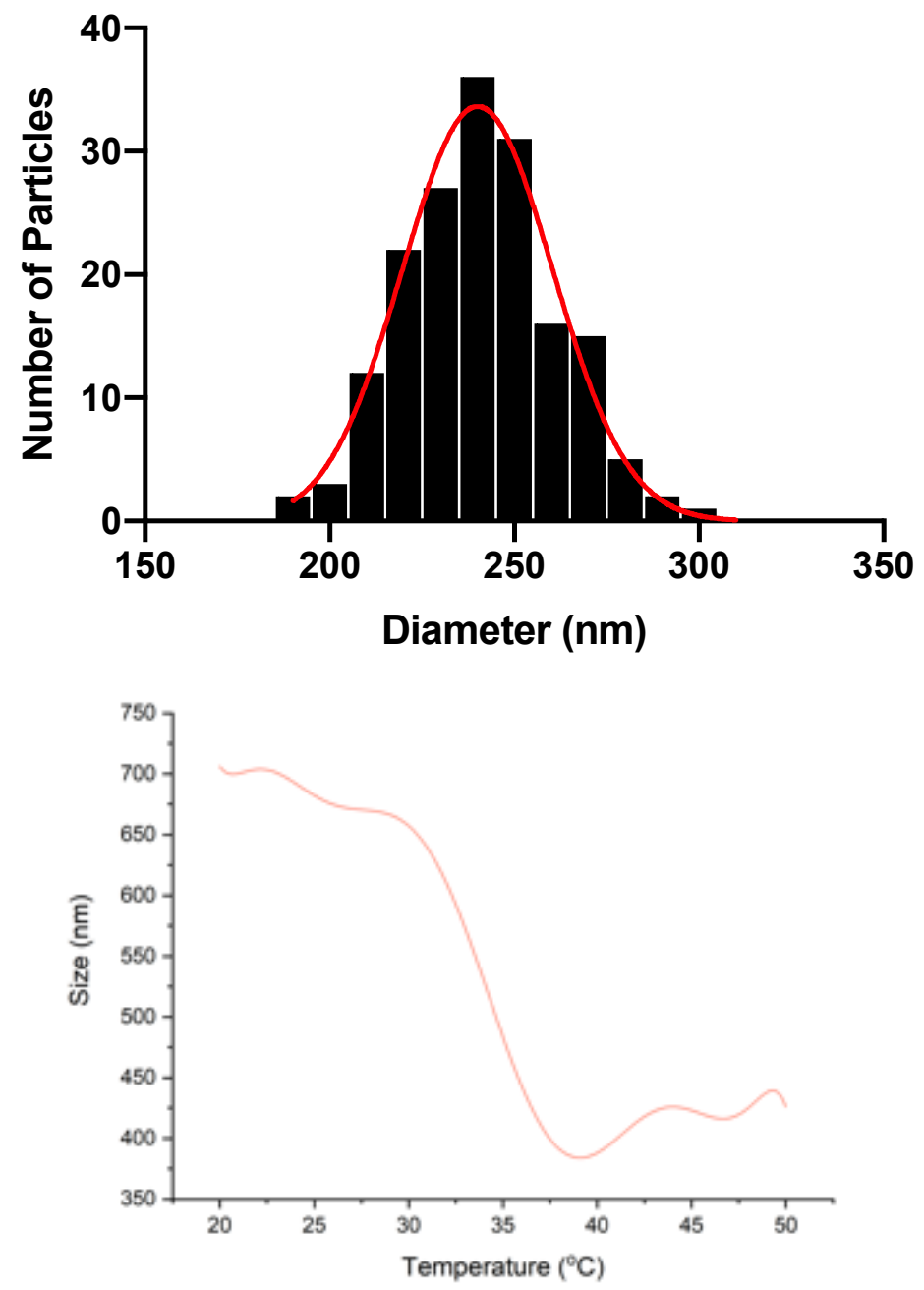

Figure 3. Size versus Temperature trend. 


\section{Cryogenic transmission electron microscopy (cryo-TEM):}
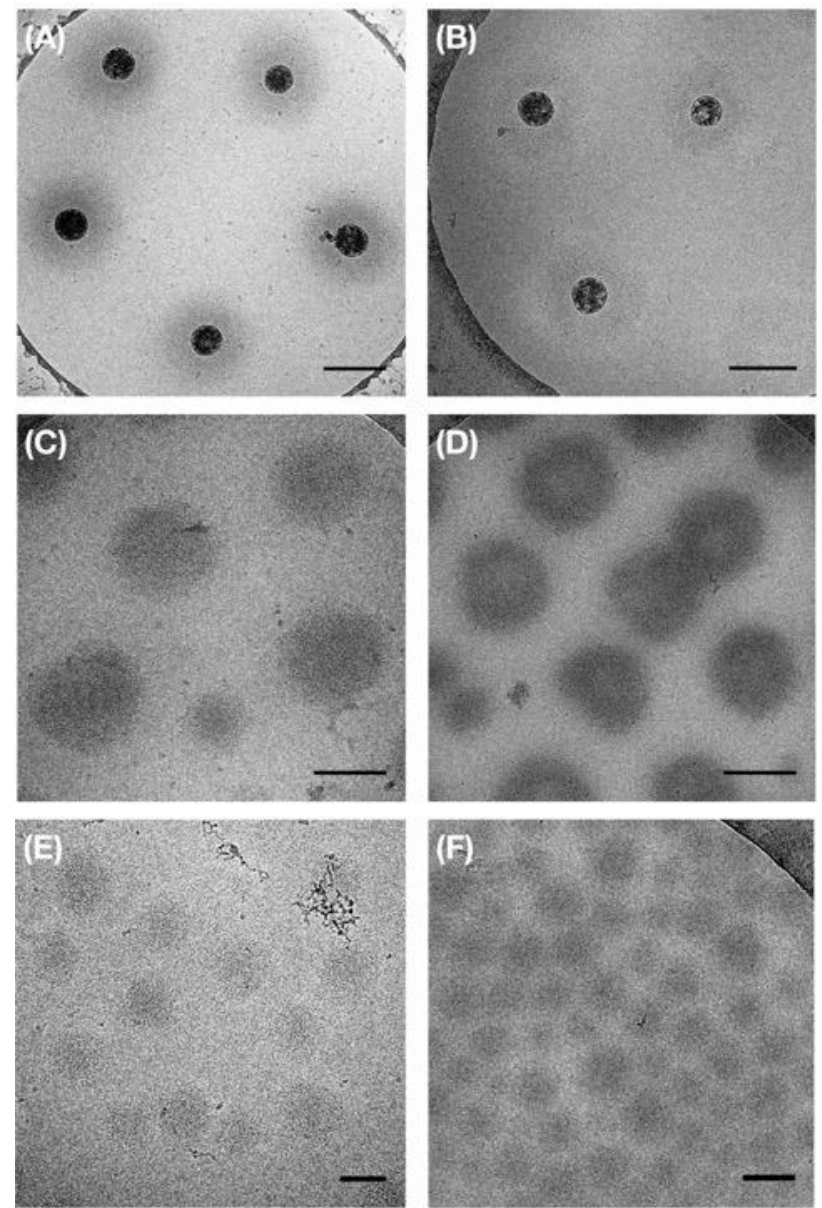

Figure 4. Cryo-TEM images of $\mathrm{SiO}_{2} @ \mathrm{NG}$ at (A) $20^{\circ} \mathrm{C}$; (B) $40^{\circ} \mathrm{C}$ and $\mathrm{NCs}$ at (C) $20^{\circ} \mathrm{C}$ and (D) $40^{\circ} \mathrm{C}$ and $\mathrm{NGs}$ at $20^{\circ} \mathrm{C}(\mathrm{E})$ and $40^{\circ} \mathrm{C}(\mathrm{F})$. Scale bar represents $200 \mathrm{~nm}(\mathrm{~A}-\mathrm{D})$ in the upper and $100 \mathrm{~nm}(\mathrm{E}$ and F) in the lower images.

\section{(A)}

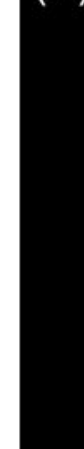

\section{(B)}

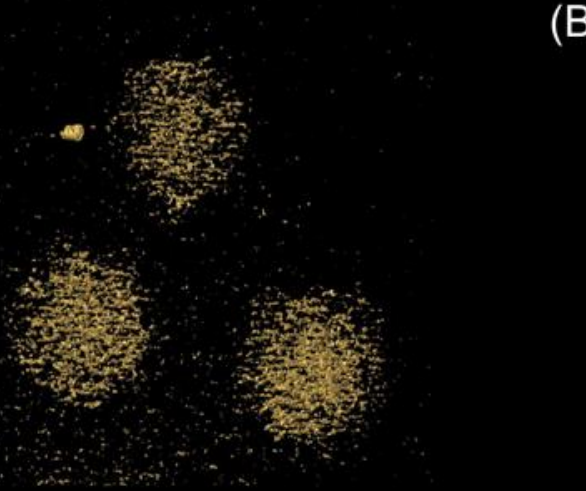

Figure 5. Isosurface representation of reconstructed cryo-ET data: (A) NGs and (B) $\mathrm{SiO}_{2} @ \mathrm{NGs}_{\text {. For better }}$ depiction of the silica core, the volume is only shown half. (visualization by AMIRA software V 6.0.0 (Thermo Fisher Scientific Inc., Waltham, Massachusetts, USA)). 


\section{PeakForce quantitative nanomechanics (QNM):}

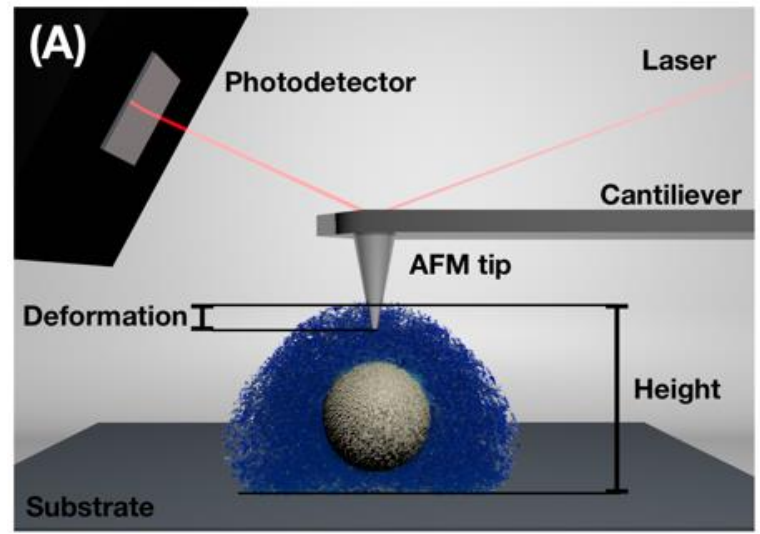

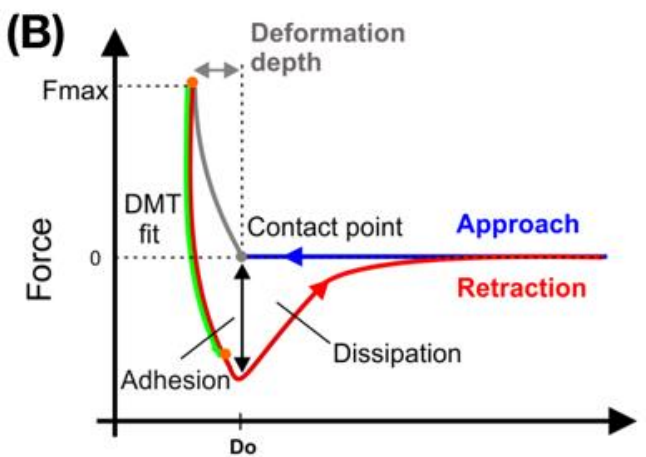

Separation distance

Figure 6. Schematic representation of the working principle of AFM in PeakForce QNM mode. (A) Key elements and parameters in the AFM tip indenting process. A very sharp tip attached at the end of a flexible cantilever indents at each point on the sample surface as the piezo is approach and retracted during a scan. If a calibrated cantilever is being used, then the vertical deflections of the cantilever can be recorded on a 4-segmented photodiode through the measurement of the reflections of a laser beam from the back of the cantilever. For the case of soft particles adhered on a hard substrate, and with a defined maximal force set point applied by the tip, a region can be scanned and the degree of deformation on soft material can be quantified. As shown in (A), deformation and height of nanoparticles can be directly measured. (B) Representation of a typical force-separation curve from which physical parameters like height, deformation, adhesion, Young's modulus and dissipation can be obtained and mapped into 2D/3D reconstruction images. As the tip approaches the sample surface, the contact point defines the beginning of the interaction between tip and sample surface for a particular force set point. From the contact point the tip compresses the sample until it reaches the force set point at $\mathrm{F}_{\max }$. If a soft sample is investigated, the separation distance between contact point and $\mathrm{F}_{\max }$, is the deformation depth by the tip. A contrast in maps of surface topography or height and deformation is expected if a soft sample (i.e. soft nanoparticles) is imaged on a hard background (substrate). In the retraction curve from model force-separation curve, further withdrawal of the AFM tip after $F_{\max }$ has been reached, provides other useful information like adhesion between tip and sample and dissipation.

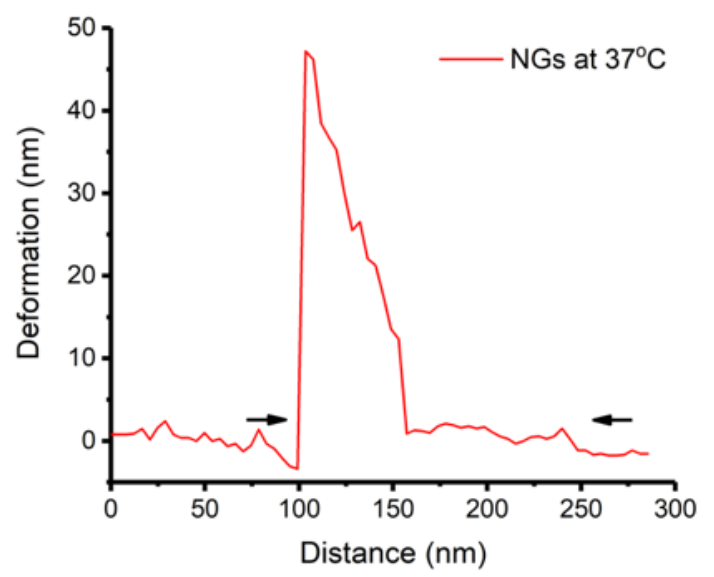

Figure 7. Cross section profile for deformation of NGs at $37{ }^{\circ} \mathrm{C}$. The cross section is taken along a different direction as the one shown in the main text in Figure 3CIV, in order to better understand the different features of deformation on the NG. From the profile, is seems that deformation by the tip increases up to almost $50 \mathrm{~nm}$ at the beginning of deformation by the tip and then suddenly drops almost entirely. The arrows indicate the lateral distance from where the NG response is obtained. A possible explanation for this, is that our $500 \mathrm{pN}$ force set point, could still be above the force threshold to damage and penetrate the NG structure. 

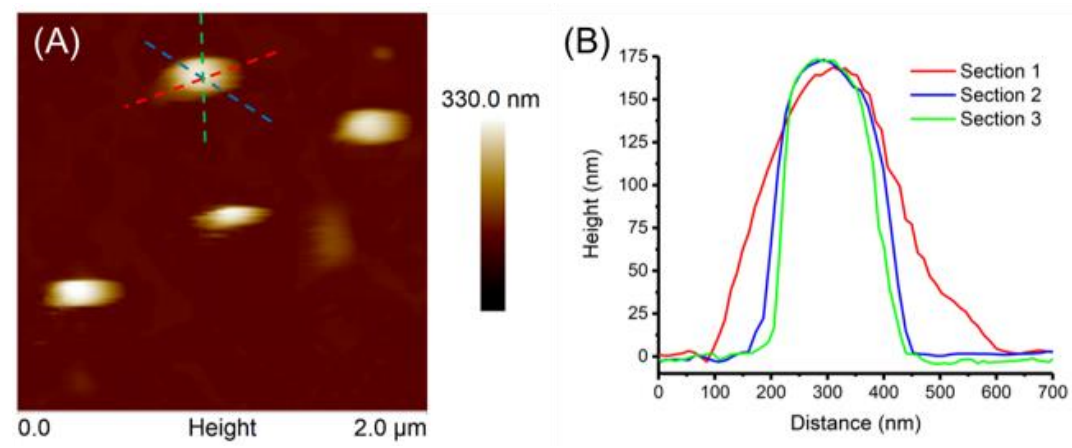

Figure 8. (A) Example of the cross-sections used for the statistic and (B) the corresponding profiles. In each particle, three cross-sections (always chosen in the same directions, two diagonal and one vertical to the mapping direction) were analyzed.

\section{Nanoindentations:}

(A)

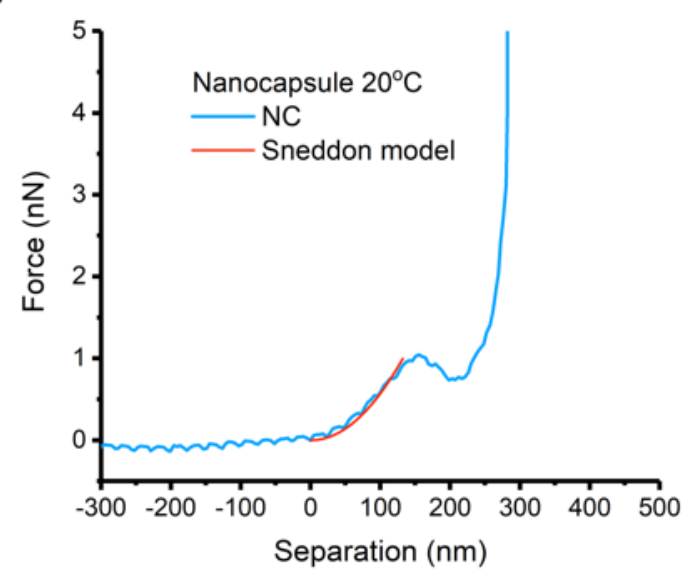

(C)

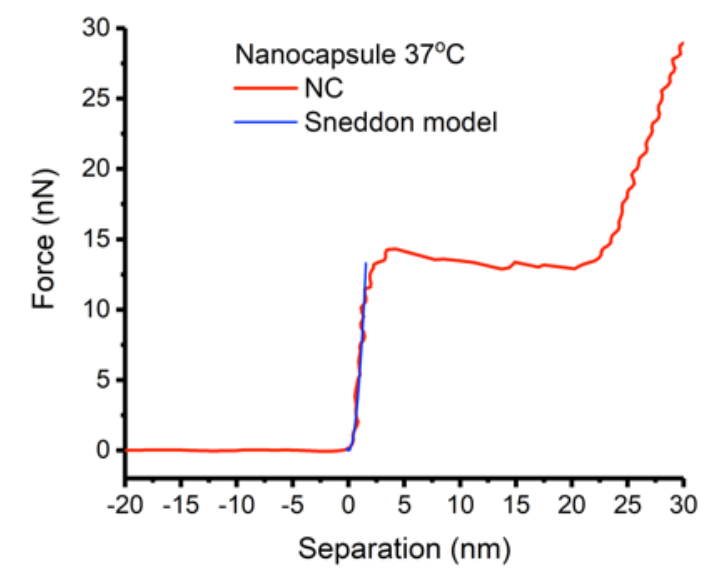

(B)

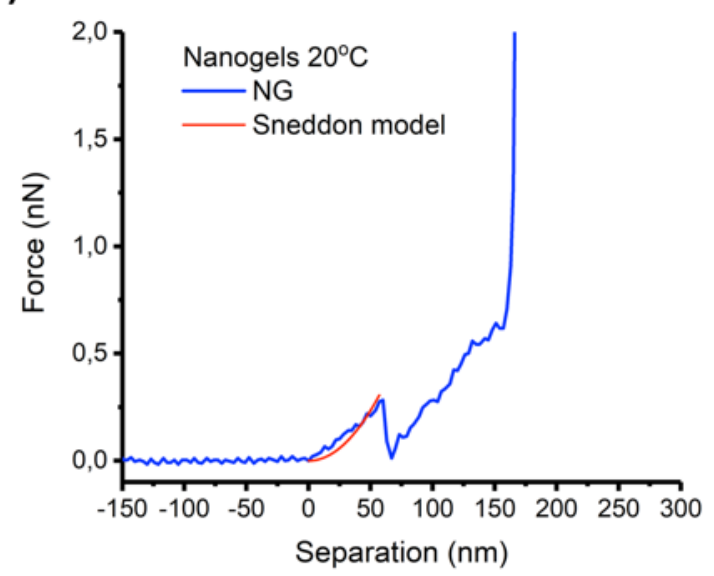

(D)

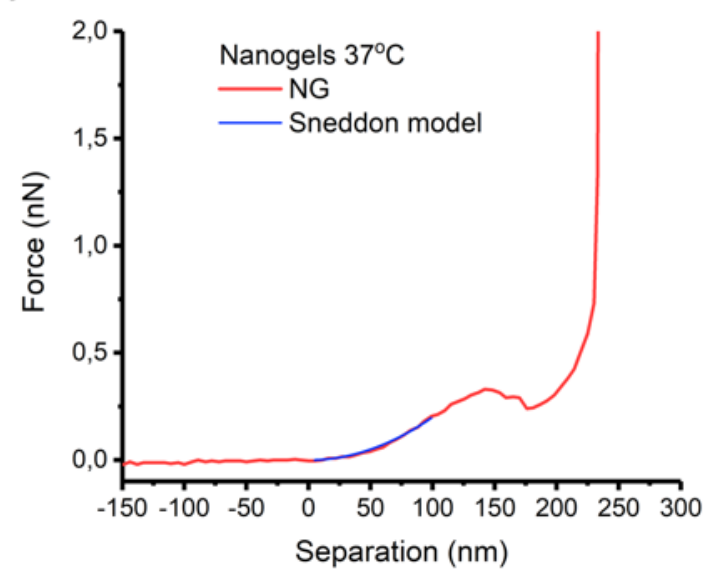

Figure 9. Force separation curves of NCs $(\mathbf{A}, \mathbf{C})$ and NGs $(\mathbf{B}, \mathbf{D})$ at $20^{\circ} \mathrm{C}$ or $37^{\circ} \mathrm{C}$ and their fit to the Sneddon model.

Finite element method: In order to describe the large displacement adequately, a distinction between current and reference frames needs to be done, as well as employing a continuum mechanical formulation suitable for providing accurate results under large deformations. We follow a well-established formulation and computational implementation based on the finite 
element method. ${ }^{3}$ All computations are established by using open-source packages and the codes are available to be used under the GNU public license.

The objective is to compute the displacement $\boldsymbol{u}$ in $\mathrm{nm}$. As units we use $\mathrm{kg}$ for mass and s for time leading to $\mathrm{nN}$ for force and GPa for stress (energy density) as well as aJ $\left(1 \mathrm{aJ}=10^{-18} \mathrm{~J}\right)$ for the energy. The displacement is a vector in space $\boldsymbol{X} \in \mathbb{R}^{3}$ and is used to compute the displacements of the continuum body occupying the region $\mathcal{B}$. By using an energy density, $\psi$ in $\mathrm{aJ} / \mathrm{nm}^{3}=\mathrm{GPa}$, the structure is modeled by a scalar function. This approach is beneficial since energy, $e$, is a measurable quantity,

$$
e=\int_{\mathcal{B}} \psi d V
$$

By using Castigliano's theorem, the force, $\boldsymbol{f}$ can be obtained, generating the displacement, $\boldsymbol{u}$, at the tip of the indenter

$$
\boldsymbol{f}=\frac{\partial e}{\partial \boldsymbol{u}} .
$$

We consider statics, i.e., the inertial effects are assumed to be negligible, since the mechanical loading is slow with respect to the response time of the material. In this case, the energy, $e$, needs to be minimized, this assertion is mathematically equivalent to constructing an action,

$$
A=\int_{\mathcal{B}} \psi d V
$$

And setting its variation to zero, $\delta A=0$. Furthermore, the deformation is induced by mechanical loading applied on the body. Related to the mechanical loading, effects of gravitational forces are negligible. The variational formulation based on the action principle in Eq. (3) can be further developed for describing the energy density by displacement. In mechanics, this energy density is called stored energy density indicating the reversible character of the underlying material. For the isothermal case, $\psi$ is equivalent to the free energy density. Neglecting the viscous character of the underlying material; an elastic, brittle response in the $\mathrm{nm}$ length scale is expected. Moreover, this concentrated loading could lead to a sharp change in strain around the contact point. Therefore, a material model accurately capturing such a sharp change is needed, herein the so-called neo-Hookean energy density is employed

$$
\psi=\frac{\lambda}{2} \ln \left(\operatorname{det}\left(F_{i j}\right)\right)^{2}+\mu\left(\frac{F_{i j} F_{i j}}{2}-\frac{\delta_{i i}}{2}-\ln \left(\operatorname{det}\left(F_{i j}\right)\right)\right), F_{i j}=\frac{\partial u_{i}}{\partial X_{j}}+\delta_{i j},
$$

With the material parameters, $\lambda, \mu$. We use standard continuum mechanical notation and Einstein's summation convention over repeated indices. The deformation gradient, $\boldsymbol{F}$, gives the relation between the energy and the displacement, $\boldsymbol{u}$, defined as a function in space, $\boldsymbol{X}$, which is tantamount to the position of material particles at the "stress free" or reference frame. The simulation is started by using a spherical nanocapsule (in a collapsed state) being at the reference frame and apply a deformation along the $x$-axis mimicking the nanoindentation experiment. The pyramid indenter tip is approximated as a conical indenter such that the distribution of displacement at the contact point---the origin set at the contact point---between the indenter tip and material body can be approximated by using a Gaussian distribution:

$$
u_{x}=d \exp \left(-\frac{\left(x^{2}+y^{2}\right)}{2 \sigma^{2}}\right) .
$$

The contact radius, $r_{c}$, is used to utilize the standard deviation, $\sigma=r_{c} / 2$. The simulation is started with a given small contact radius that is updated depending on the indentation depth, $d$, by means of the geometric relation for a cone, $r_{c}=d \tan (\alpha)$, with the constant tip angle set as $\alpha=20^{\circ}$ based on the specification of the indenter tip. The amplitude of the Gaussian distribution is set analogous to the experiments, in $60 \mathrm{~s}$ the amplitude reaches $10 \mathrm{~nm}$ : Implementation and computation are very challenging in the case of a so-called point force. The proposed distribution is an accurate model. For the description of the energy density in Eq. (4), the so-called Lame constants are used

$$
\lambda=\frac{E v}{(1+v)(1-2 v)}, \mu=\frac{E}{2(1+v)},
$$

They are related to the engineering constants: Young's modulus, $E$, and Poisson's ratio, $v$. $E=$ $17.1 \mathrm{GPa}$ and $v=0.45$ are used in order to mimic the experimental results (the used values are 
the result from the nanoindentation of the representative NCs shown in the main article Figure 5C). Young's modulus is obtained with the aid of the Sneddon's solution and we expect to be able to justify the simplifications in this solution by comparing the computational and experimental results.

In order to solve $\delta A=0$ with Eq. (3), the finite element method (FEM) is used with the aid of open-source packages developed under FEniCS project. Geometry is constructed in Salome and its triangulation is established by NetGen algorithm. Only a quarter of the spherical is used owing to the symmetry conditions. In Figure 10, the geometry and the regions for the symmetry conditions are shown.

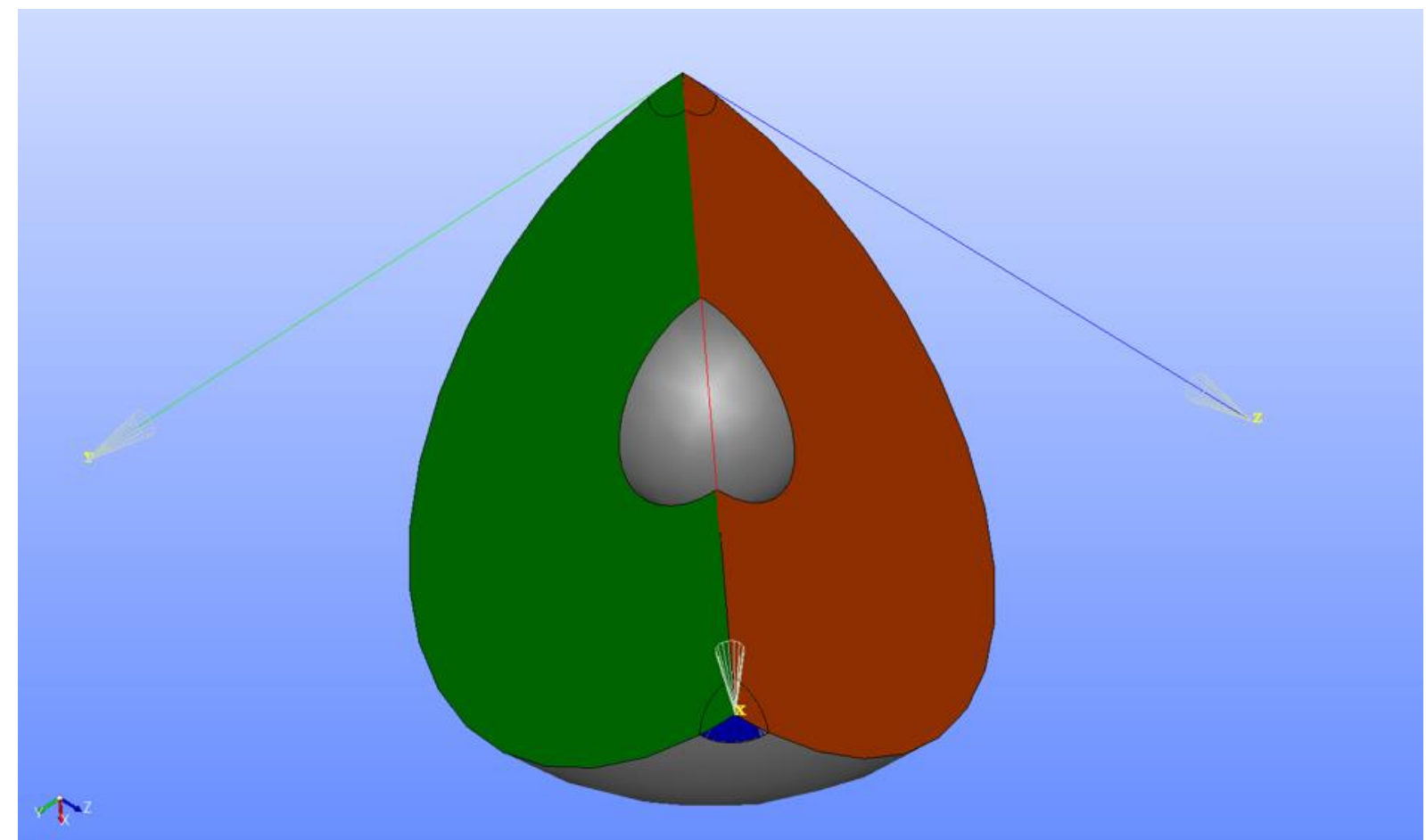

Figure 10. Geometry constructed and visualized in Salome. The quarter of the nanocapsule is simulated by applying symmetric boundary conditions along $x$ on blue, along $y$ on orange and along $z$ on green surfaces.

The mesh out of tetrahedron elements are generated as in Figure 11. Around the contact point of the indentation tip, a gradually finer mesh is utilized.
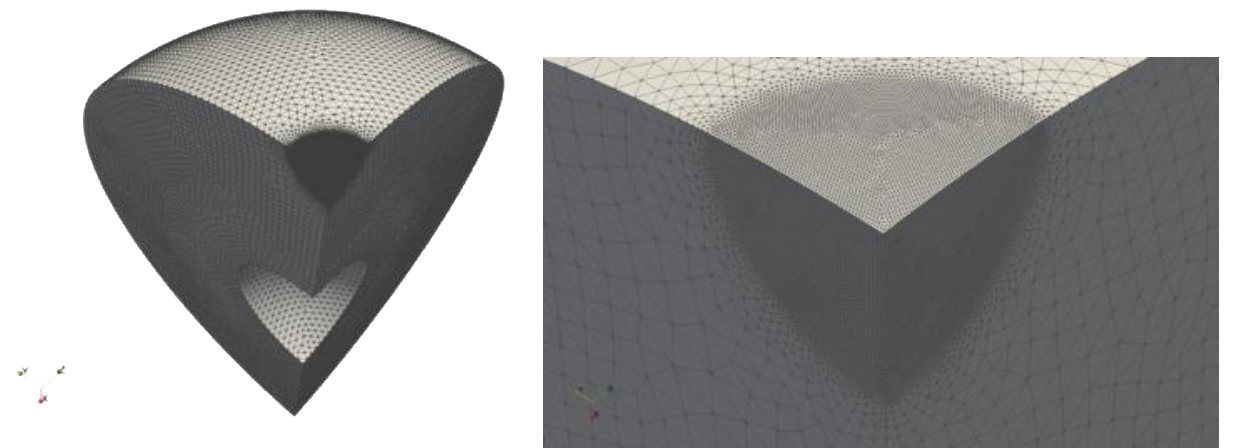

Figure 11. Mesh is generated by NetGen in Salome and visualized in ParaView. Left: computational domain. Right: a close up showing the finer mesh around the nanoindentation.

The computation of 429531 degrees of freedom has been established in parallel on 6 Intel Xeaon E7-4850 v4 on Supermicro running Ubuntu Operating System in 2 hours of computation time by using a conjugate gradient type iterative solver with an algebraic multigrid preconditioner. For the simulation of $60 \mathrm{~s}$, a time step of $0.5 \mathrm{~s}$ is chosen. Up to $10 \mathrm{~nm}$ is indented 
by using the aforementioned distribution in Eq. (5). The displacement at the final time is visualized (without scaling) in Figure 12 by using ParaView.

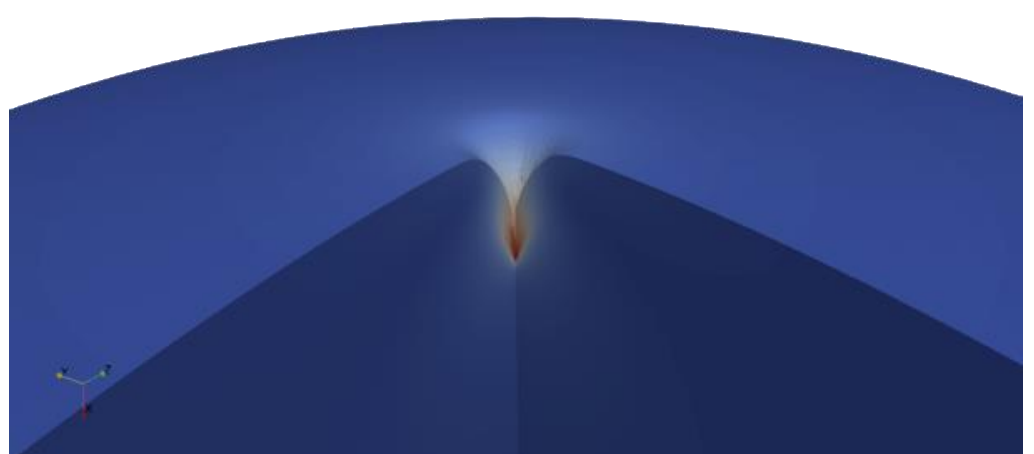

Figure 12. Displacement at the final time with amplitude of $10 \mathrm{~nm}$. The close up around the origin is shown in ParaView by mirroring the solution for the sake of a better visualization. The colors denote the magnitude of displacement, whereas the displacement is shown without scaling.

The energy in the whole body is computed for each time step; the force at the contact point is extracted out of the energy by using Eq. (2).

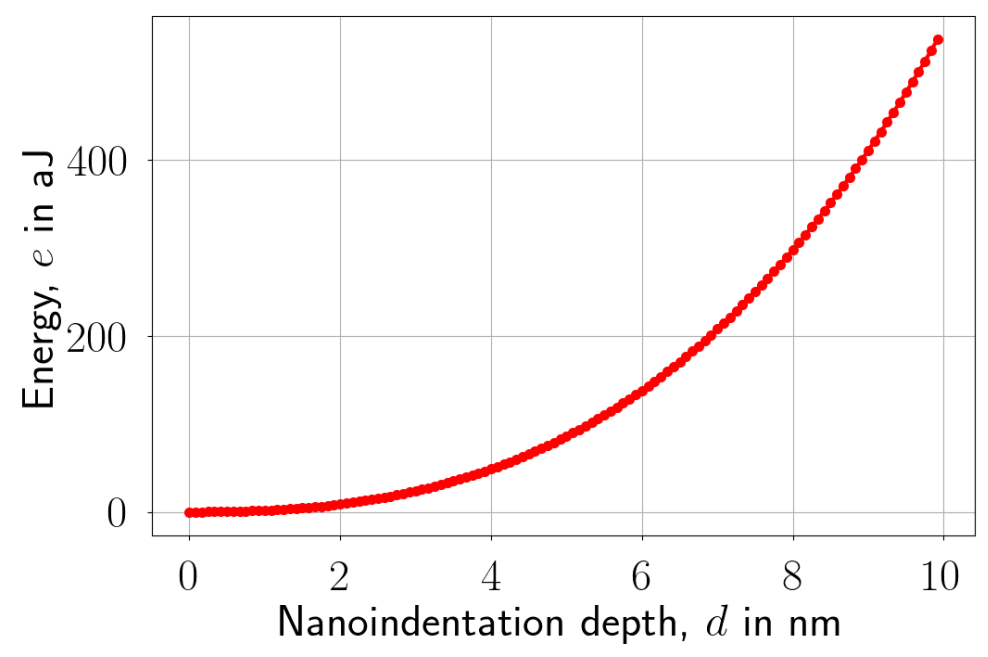

Figure 13. Energy versus nanoindentation depth.

1. Li, S.; Wang, J.; Zhao, S.; Cai, W.; Wang, Z.; Wang, S., Effect of surface modification and medium on the rheological properties of silica nanoparticle suspensions. Ceramics International 2016, 42 (6), 7767-7773.

2. Dubbert, J.; Nothdurft, K.; Karg, M.; Richtering, W., Core-shell-shell and hollow double-shell microgels with advanced temperature responsiveness. Macromol Rapid Commun 2015, 36 (2), 159-64.

3. Abali, B. E., Computational Reality, Solving Nonlinear and Coupled Problems in Continuum Mechanics. Advanced Structured Materials, Springer Nature, Singapore: 2017; Vol. 55. 